\title{
Cluster Size Effect of X-Ray Fluorescence Hologram Simulation Using $\mathrm{Sr}_{0.95} \mathrm{La}_{0.05} \mathrm{TiO}_{3}$
}

\author{
Yoshihiro Ebisu $^{1 *}$, Tomohiro Matsushita ${ }^{2}$, Naohisa Happo ${ }^{3}$ and Tōru Ozaki ${ }^{1}$ \\ 1 Graduate School of Science and Technology, Hiroshima Institute of Technology, Hiroshima 731-5193, Japan \\ 2 Japan Synchrotron Radiation Research Institute, Hyogo 679-5198, Japan \\ 3 Graduate School of Information Sciences, Hiroshima City University, Hiroshima 731-3194, Japan \\ * Corresponding author: e-mail: d111501@cc.it-hiroshima.ac.jp and ozaki@cc.it-hiroshima.ac.jp
}

\begin{abstract}
We simulated $L a \gamma_{1}$ X-ray fluorescence holography ( $\mathrm{La} L \gamma_{1} \mathrm{XFH}$ ) on a spherical cluster model of $\mathrm{Sr}_{0.95} \mathrm{La}_{0.05} \mathrm{TiO}_{3}$. As the radius of the model $r_{\mathrm{c}}$ increased from $10 \AA$ to $200 \AA$, the simulated hologram pattern became finer and sharper. The X-ray standing wave lines in the simulated hologram of the model with $r_{\mathrm{c}}=200 \AA$ reproduced those in the experimentally obtained hologram well. Because the X-ray fluorescence hologram is defined as a function on a spherical surface, we estimate the fineness of the pattern by calculating the power spectrum with the spherical harmonics transform. The power spectrum of the holographic oscillations of the experimentally obtained hologram shows no cutoff below the Nyquist frequency. The power spectra of the models with $60 \AA \leq r_{\mathrm{c}} \leq 200 \AA$ do not show cutoffs either. These indicate that the radius of the cluster model $r_{\mathrm{c}}$ should be set at least $200 \AA$ to reproduce the experimentally obtained hologram of $\mathrm{Sr}_{0.95} \mathrm{La}_{0.05} \mathrm{TiO}_{3}$.
\end{abstract}

Key words: $\mathrm{Sr}_{0.95} \mathrm{La}_{0.05} \mathrm{TiO}_{3}$, X-ray fluorescence holography, holographic oscillation, power spectrum

\section{INTRODUCTION}

$\mathrm{X}$-ray fluorescence holography $(\mathrm{XFH})$ is a powerful method for determining three-dimensional (3D) local atomic arrangements around a specific fluorescent atom. $\mathrm{XFH}$ with synchrotron X-rays allow for atomic images of thin films [1-3] and doped crystals [4, 5] containing a small amount of dopant to be obtained. The physical properties of the substances have been investigated based on the 3D local atomic structures. The advantage of the XFH measurement is that the 3D local atomic structures are directly reconstructed without any model in principle [6]. In practice, however, the simulation of XFH is often performed on cluster models to confirm the experimentally obtained local structures. The models are of radii $r_{\mathrm{c}}$ ranging from $20 \AA$ to $60 \AA$ around the fluorescent atoms and can reproduce the experimentally obtained local structures [4, 5, 7-12]. However, it is not investigated to obtain the appropriate size $r_{\mathrm{c}}$ of the cluster model.

Recently, we performed the La $L \gamma_{1}$ XFH of $\mathrm{Sr}_{0.95} \mathrm{La}_{0.05} \mathrm{TiO}_{3}$ to investigate the local structure around the La atom [4]. The local structure showed that the La atoms were substituted for the $\mathrm{Sr}$ atoms in the cubic perovskite structure of $\mathrm{SrTiO}_{3}$, as illustrated in Fig. 1 . The atomic distance between the $\mathrm{La}$ atom and the nearest neighbor $\mathrm{Sr}(\mathrm{La})$ atom is slightly larger than that between the $\mathrm{Sr}$ atoms in $\mathrm{SrTiO}_{3}$. We also simulated the La $L \gamma_{1}$ XFH of a spherical cluster model with the radius of $r_{\mathrm{c}}=50 \AA$. The calculated holograms were similar to those obtained experimentally. However, we could not confirm the appropriate cluster size. Herein, we numerically investigate the cluster size dependence of the hologram pattern and the fineness of the holographic oscillations using the standing wave lines and spherical harmonics transform.

\section{SIMULATION}

2.1 Simulation of X-ray fluorescence hologram

We prepared a spherical atomic cluster model of $\mathrm{Sr}_{0.95} \mathrm{La}_{0.05} \mathrm{TiO}_{3}$ with radius $r_{\mathrm{c}}$, as illustrated in Fig.1. The model has a La atom on the origin of the coordinate axes $x, y$ and $z$ of the cubic perovskite structure [4]. The atomic form factors $0.95 f_{\mathrm{Sr}}+0.05 f_{\mathrm{La}}$ are set to the $\mathrm{Sr}(\mathrm{La})$ atoms. This describes that a $\mathrm{La}$ atom is substituted for a

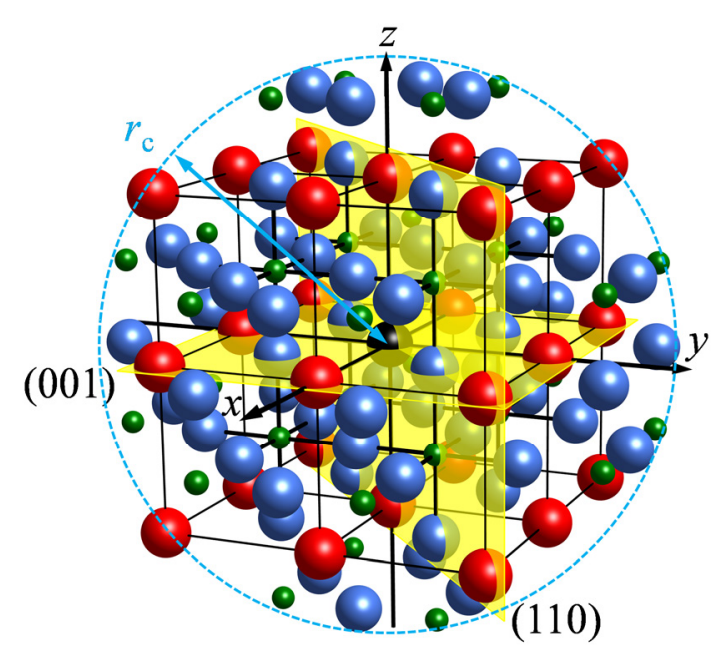

Fig. 1 Spherical cluster model of $\mathrm{Sr}_{0.95} \mathrm{La}_{0.05} \mathrm{TiO}_{3}$ with radius $r_{\mathrm{c}}$. The black sphere on the origin of the coordinate axes $x, y$, and $z$ represents the La atom. The red ones are the $\operatorname{Sr}(\mathrm{La})$ atoms with the atomic form factors of $0.95 f_{\mathrm{Sr}}+0.05 f_{\mathrm{La}}$. The blue ones and the green ones are the $\mathrm{O}$ atoms and the $\mathrm{Ti}$ atoms, respectively. The yellow planes indicate the (001) and the (110) planes. 
$\mathrm{Sr}$ atom randomly with the probability of 0.05 . The value of the lattice parameter is $3.907 \AA$, which was measured with the Bond method at $295 \mathrm{~K}$. Thus, we created eleven spherical cluster models of $\mathrm{Sr}_{0.95} \mathrm{La}_{0.05} \mathrm{TiO}_{3}$ with $r_{\mathrm{c}}=10,20,30,40,50,60,70,80$, 90,100 , and $200 \AA$.

We simulated the La $L \gamma_{1}$ XFH of each cluster model of $\mathrm{Sr}_{0.95} \mathrm{La}_{0.05} \mathrm{TiO}_{3}$ in the single scattering case of the inverse mode. Figure 2 describes the interference between the incident and scattered X-rays on the La atom located on the origin of the coordinate axes $x, y$, and $z$. The linearly polarized X-ray having the wave vector $\boldsymbol{k}$ and the electric field $\boldsymbol{E}$ normal to the $y$ axis is incident on the $\mathrm{La}$ atom. We fixed the direction of the incident X-ray and rotated the $\mathrm{Sr}_{0.95} \mathrm{La}_{0.05} \mathrm{TiO}_{3}$ cluster model around the $z$ and the $x$ axes of the azimuthal angle $\phi$ and the polar angle $\theta$, similarly as in the previous experiment [4]. The incident X-ray is also scattered by the $j$ th atom located at $\boldsymbol{r}_{j}$. The scattered X-ray incident on the La atom has the wave vector $\boldsymbol{k}_{j}$ and the electric field $\boldsymbol{E}_{j}$ with the scattering angle $\Theta_{j}$. The incident and scattered $\mathrm{X}$-rays interfere on the La atom and excite the La $L \gamma_{1}$ fluorescent X-ray. Consequently, the holographic oscillation of the $j$ th atom appears on the fluorescent X-ray intensity depending on $\Theta_{j}$. The hologram function is given by the following equation [13],

$$
\begin{aligned}
& \chi(\boldsymbol{k})=\sum_{j} \chi_{j}(\boldsymbol{k}) \\
& =-2 r_{\mathrm{e}} \sum_{j} \frac{f_{j}\left(k, \Theta_{j}\right)}{r_{j}} P_{j} \cos \left(-k r_{j} \cos \Theta_{j}+k r_{j}\right),
\end{aligned}
$$

where $r_{\mathrm{e}}$ represents the classical electron radius, $r_{j}$ the distance of the $j$ th atom from the La atom, and $P_{j}=$ $P_{j}(\theta, \phi)$ is the polarization factor. The atomic form factor $f_{j}\left(k, \Theta_{j}\right)$ depends on the scattering angle $\Theta_{j}=\Theta_{j}(\theta, \phi)$, as illustrated in Fig. 2. Equation 1 indicates that the measured hologram is given by the simple sum of the hologram of each atom.

To reproduce the experimental geometry [4], the value of $\chi(\boldsymbol{k})$ in Eq. 1 was calculated in the range of $0^{\circ} \leq$ $\phi \leq 360^{\circ}$ in steps of $\Delta \phi=1.00^{\circ}$ and in the limited range of $0^{\circ} \leq \theta \leq 75^{\circ}$ in steps of $\Delta \theta=1.00^{\circ}{ }^{*}$ The $4 \pi \mathrm{sr}$ hologram was calculated by the symmetry operation according to the symmetry elements of the cubic perovskite structure, the fourfold rotation axes parallel to the [001], the [010] and [100] axes, and the mirror plane parallel to the (100) plane.

Figure 3 shows the holograms of the cluster models with $r_{\mathrm{c}}=10,20,30,40,50,100$, and $200 \AA$. The incident X-ray energy is $7.00 \mathrm{keV}$. The holograms are described by the orthographic projection. As the radius of the cluster model $r_{\mathrm{c}}$ increases from $10 \AA$ to $200 \AA$, the amplitude of the holographic oscillation becomes larger and the hologram pattern becomes finer. The X-ray standing wave lines, which are generated by the reflection on the atomic planes, appear above $r_{\mathrm{c}}=30 \AA$. One of the $\{110\} X$-ray standing wave lines is indicated between a pair of light blue arrows in Fig. 3(g).

\footnotetext{
* The measurement was carried out in steps of $\Delta \theta=$ $1.00^{\circ}$ and $\Delta \phi=0.300^{\circ}$. And then, we resampled the data in steps of $\Delta \phi=1.00^{\circ}$ to align the step sizes of $\theta$ and $\phi$.
}

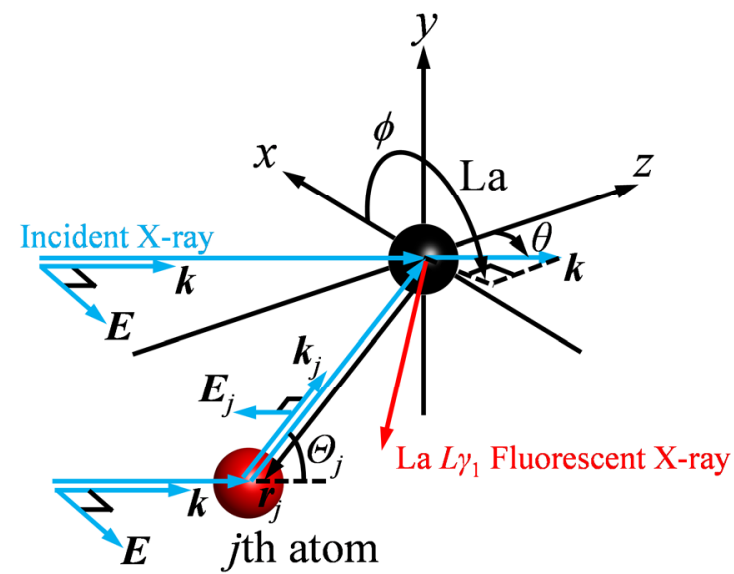

Fig. 2 Schematic to describe the interference between the incident and scattered X-rays and the emission of the La $L \gamma_{1}$ fluorescent X-ray.

The corresponding indexes of the reflections $\{h k l\}$ of the X-ray standing wave lines are described in Fig. 4. Compared with Fig. 3, the $\{110\}$ X-ray standing wave lines colored by light blue become sharper above $r_{\mathrm{c}}=30$ $\AA$ and the $\{331\}$ X-ray standing wave lines colored by violet become sharper above $r_{\mathrm{c}}=100 \AA$.

The hologram measured at the incident X-ray energy of $7.00 \mathrm{keV}$ at $T=297 \mathrm{~K}$ is also shown for the comparison in Fig. 3(h) [4]. The $\{331\}$ X-ray standing wave lines are as sharp as those of the model with $r_{\mathrm{c}}=$ $200 \AA$. Thus, the pattern of the measured hologram resembles that of the calculated hologram with $r_{\mathrm{c}}=200$ $\AA$. This indicates that the X-rays scattered by the atoms located further than $200 \AA$ from the La atom interfere with the incident X-ray on the La atom. To evaluate the fineness of the measured and calculated hologram patterns, we performed a power spectrum analysis.

\subsection{Power spectrum of holographic oscillation}

The power spectrum of the holographic oscillation was calculated from the hologram pattern shown in Fig. 3 using the spherical harmonics transform. The holographic oscillation $\chi(k, \theta, \phi)$ can be expressed by the sum of the spherical harmonics $\mathrm{Y}_{l}^{m}(\theta, \phi)$,

$$
\chi(k, \theta, \phi)=\sum_{l=0}^{\infty} \sum_{m=-l}^{l} A_{l}^{m} Y_{l}^{m}(\theta, \phi),
$$

where $l$ is a natural number and $m$ is an integer. The expansion coefficient $A_{l}^{m}$ is obtained by the spherical harmonics transform,

$$
A_{l}^{m}=\int_{0}^{2 \pi} \int_{0}^{\pi} \chi(k, \theta, \phi) \mathrm{Y}_{l}^{m}(\theta, \phi) \sin \theta \mathrm{d} \theta \mathrm{d} \phi .
$$

This is similar to the Fourier transform. Therefore, the power spectrum of the holographic oscillation $S(l)$ is expressed by the sum of $\left(A_{l}^{m}\right)^{2}$,

$$
S(l)=\sum_{m=-l}^{l}\left(A_{l}^{m}\right)^{2} .
$$




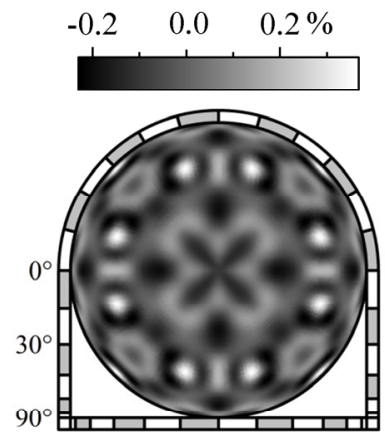

(a) $r_{\mathrm{c}}=10 \AA$

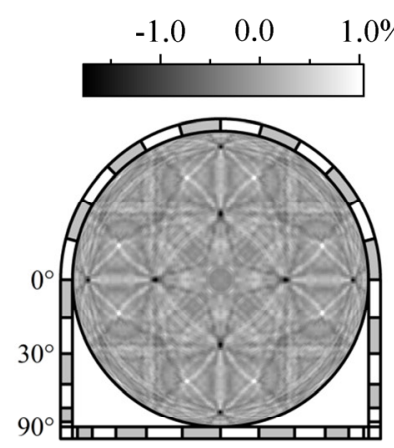

(e) $r_{\mathrm{c}}=50 \AA$

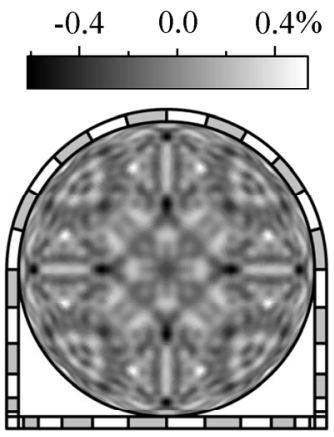

(b) $r_{\mathrm{c}}=20 \AA$

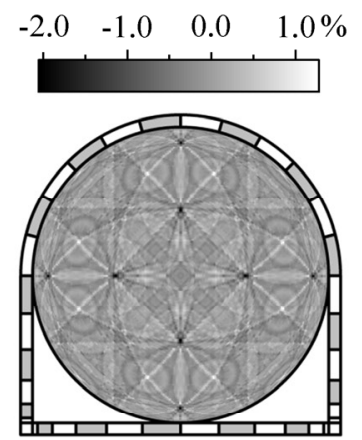

(f) $r_{\mathrm{c}}=100 \AA$

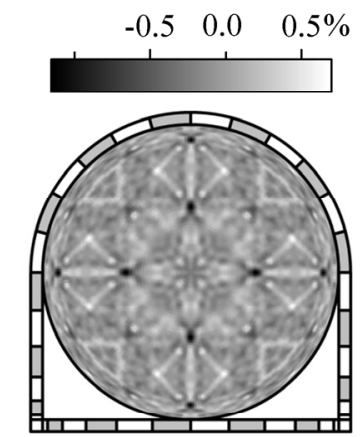

(c) $r_{\mathrm{c}}=30 \AA$

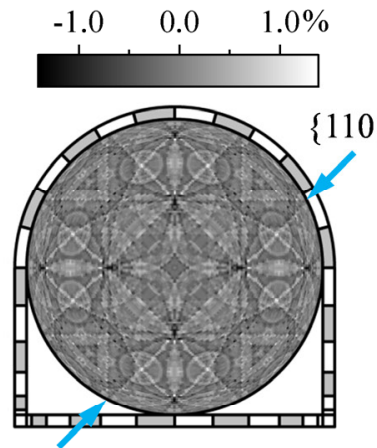

(g) $r_{\mathrm{c}}=200 \AA$

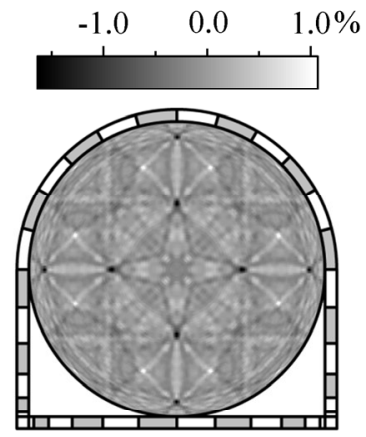

(d) $r_{\mathrm{c}}=40 \AA$

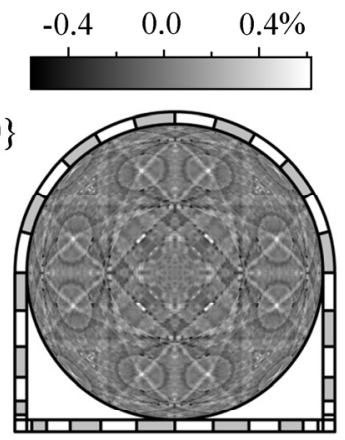

(h) Measured

Fig. 3 Atomic cluster size dependence of the hologram pattern of $\mathrm{Sr}_{0.95} \mathrm{La}_{0.05} \mathrm{TiO}_{3}$ at the incident X-ray energy of 7.00 $\mathrm{keV}$. The value of the radius of the cluster model is (a) $r_{\mathrm{c}}=10 \AA$, (b) $r_{\mathrm{c}}=20 \AA$, (c) $r_{\mathrm{c}}=30 \AA$, (d) $r_{\mathrm{c}}=40 \AA$, (e) $r_{\mathrm{c}}=50 \AA$, (f) $r_{\mathrm{c}}=100 \AA$, and (g) $r_{\mathrm{c}}=200 \AA$. The hologram measured at $7.00 \mathrm{keV}$ and at $297 \mathrm{~K}$ is shown for the comparison in (h) Measured [4]. The scale of the holographic oscillation is represented in each hologram. A pair of arrows in the hologram (g) holds one of the $\{110\} \mathrm{X}$-ray standing wave lines.

The spherical harmonics $\mathrm{Y}_{l}^{m}(\theta, \phi)$ oscillates $n$ times per a degree of $\theta, n=(l / 2) / 180\left[\mathrm{deg}^{-1}\right]$. The Nyquist frequency, which is the upper limit of the frequency interval, is $n=0.500 \mathrm{deg}^{-1}$ which is equal to a half of the sampling frequency $1 / \Delta \theta=1.00 \mathrm{deg}^{-1}$ and yields the maximum size of $\mathrm{Y}_{l}^{m}(\theta, \phi), l=180$. Therefore, it is to calculate $S(l)$ in Eq. 4 in the range of $0 \leq l \leq 180$.

The atomic cluster size dependence of the power spectrum $S(l)$ is shown in Fig. 5. The $S(l)$ shows the increase in the range of $0 \leq l \leq 20$ at all $r_{\mathrm{c}}$. In the range of $20 \leq l \leq 180$, the $S(l)$ show the cutoffs at $10 \AA \leq r_{\mathrm{c}} \leq$ $50 \AA$, of which the cutoff positions $l_{\text {cut }}$ are pointed by the arrows in the figure. The $l_{\text {cut }}$ have the values around 35 at $r_{\mathrm{c}}=10 \AA, 70$ at $r_{\mathrm{c}}=20 \AA, 100$ at $r_{\mathrm{c}}=30 \AA, 140$ at $r_{\mathrm{c}}=$ $40 \AA$, and 175 at $r_{\mathrm{c}}=50 \AA$. Thus the $l_{\text {cut }}$ is proportional to the $r_{\mathrm{c}}$ according to the relation $l_{\text {cut }}=k r_{\mathrm{c}}$ at $k=3.55 \AA^{-1}$, which is derived from Eqs. 1 and 2. This indicates that the $l_{\text {cut }}$ corresponds to the highest frequency of the holographic oscillations of the atoms at the furthest positions from the center of each cluster with $r_{\mathrm{c}}$. Therefore, all of the atoms in each cluster model with $r_{\mathrm{c}}$ $\leq 50 \AA$ show the holographic oscillations in the range of $l \leq l_{\text {cut }}$ in Fig. 5. At $r_{\mathrm{c}} \geq 60 \AA$, on the other hand, $S(l)$ show no cutoffs in the range of $l \leq 180$. At $r_{\mathrm{c}}=60,80$, $100 \AA, S(l)$ show the slight decreases in the range of 110 $\leq l \leq 180$. These indicate that the $S(l)$ at $r_{\mathrm{c}} \geq 60 \AA$ include the holographic oscillations higher than the Nyquist frequency in the range of $l \geq 180$.
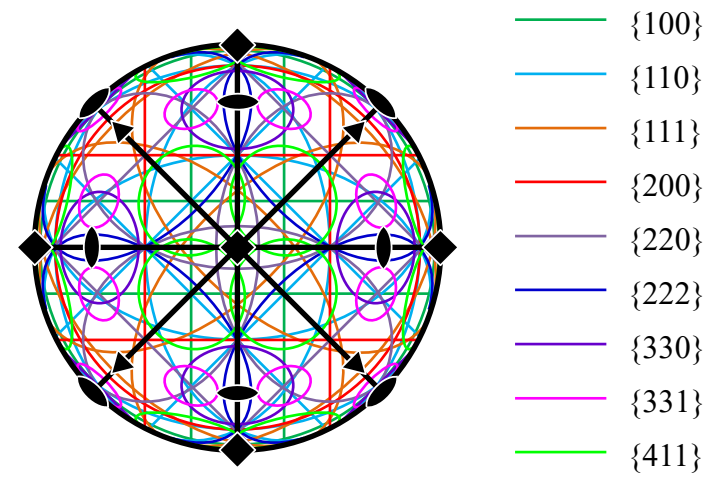

Fig. $4 \mathrm{X}$-ray standing wave lines and symmetry elements observed in the $\mathrm{La} L \gamma_{1} \mathrm{X}$-ray fluorescence hologram of the $\mathrm{Sr}_{0.95} \mathrm{La}_{0.05} \mathrm{TiO}_{3}$ cluster model with $r_{\mathrm{c}}=200 \AA$ in Fig. $3(\mathrm{~g})$. The incident X-ray energy is $7.00 \mathrm{keV}$. The index of the reflection $\{h k l\}$ is specified by the color of each line. The directions of the fourfold rotation axes, threefold rotation axes, twofold rotation axes, and the orientations of the mirror planes are indicated by their symbols. 


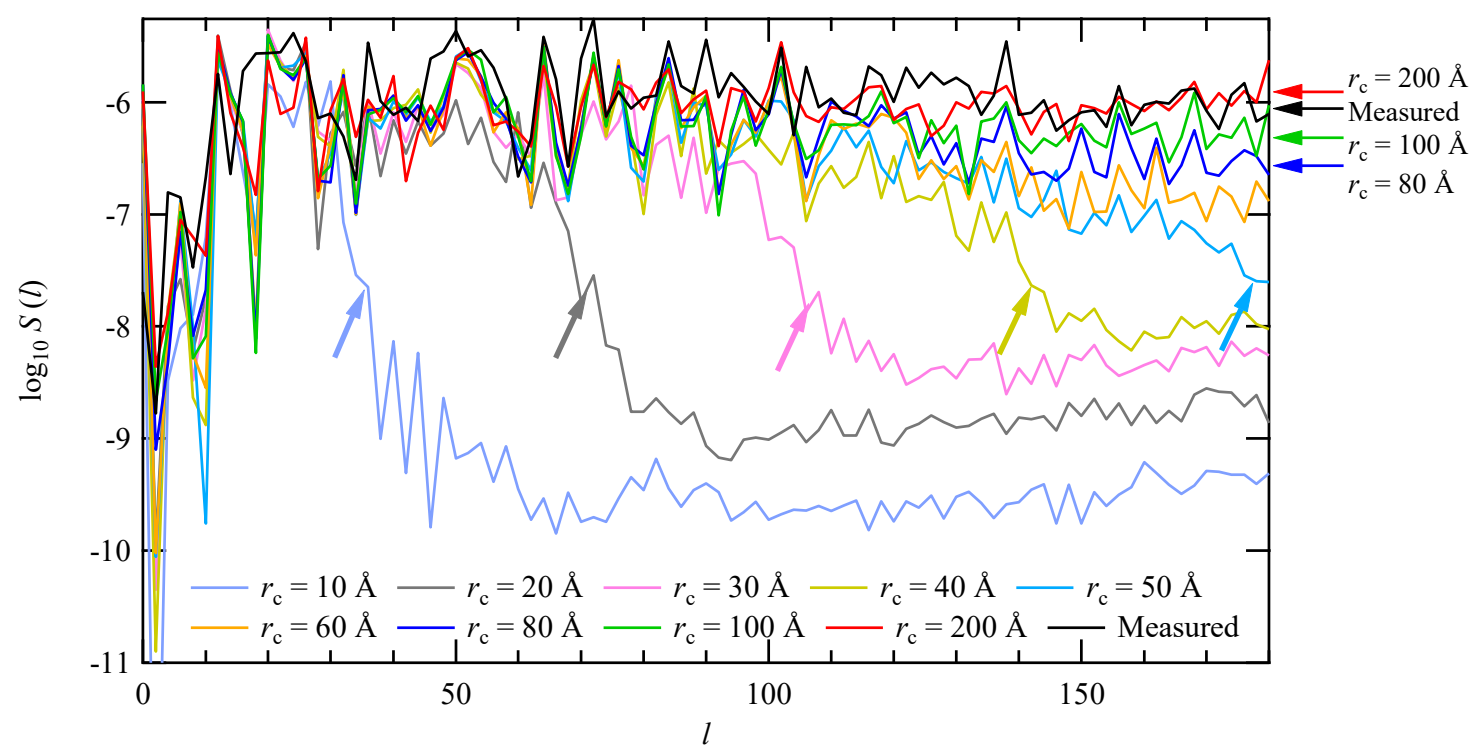

Fig. 5 Power spectrum $S(l)$ of X-ray fluorescence holographic oscillations of the $\mathrm{Sr}_{0.95} \mathrm{La}_{0.05} \mathrm{TiO}_{3}$ cluster model with the radius $r_{\mathrm{c}}$ ranging from $10 \AA$ to $200 \AA$. Each arrow points the cutoff position $l_{\text {cut }}$ at each $r_{\mathrm{c}}$. The power spectrum $S(l)$ of the holographic oscillations measured at $297 \mathrm{~K}$ is also shown. The incident X-ray has the wavenumber $k=3.55 \AA^{-1}$.

The $S(l)$ of the measured hologram is shown for comparison in Fig. 5. The values of the measured $S(l)$ are normalized with those of the calculated $S(l)$ of the models with $r_{\mathrm{c}}$ larger than $30 \AA$ at $l=53$. The measured $S(l)$ contains no cutoff but exhibits the value equal to that of $r_{\mathrm{c}}=200 \AA$ in the range of $0 \leq l \leq 180$. Therefore, the power spectra indicate that the measured $S(l)$ is evaluated quantitatively by the calculated $S(l)$ at $r_{\mathrm{c}}=200$ $\AA$. This supports that the measured hologram pattern is as fine as the calculated one of the model with $r_{\mathrm{c}}=200$ $\AA$ in Fig. 3.

\section{Conclusion}

We calculated the $\mathrm{La} L \gamma_{1} \quad \mathrm{X}$-ray fluorescence holographic oscillations $\chi(\boldsymbol{k})$ of a spherical atomic cluster model of $\mathrm{Sr}_{0.95} \mathrm{La}_{0.05} \mathrm{TiO}_{3}$ by changing the cluster size. As the radius of the cluster model $r_{\mathrm{c}}$ increased from $10 \AA$ to $200 \AA$, the hologram pattern became finer and sharper similar to that of the measured hologram pattern in Fig. 3. The X-ray standing wave lines observed in the measured hologram resembled those of the calculated hologram of the model with $r_{\mathrm{c}}=200 \AA$. Using the spherical harmonics transform, the $S(l)$ of the holographic oscillations were calculated below the Nyquist frequency of $0.500 \mathrm{deg}^{-1}$ in the range of $0 \leq l \leq$ 180. $S(l)$ shows the cutoffs in the models with $10 \AA \leq r_{\mathrm{c}}$ $\leq 50 \AA$, but no cutoffs in the models with $60 \AA \leq r_{\mathrm{c}} \leq$ $200 \AA$.

The $S(l)$ of the measured hologram had no cutoff but exhibited good agreement with that of the simulated hologram of the $200 \AA$ cluster. Therefore, to simulate the X-ray fluorescence hologram precisely, the cluster radius of $\mathrm{Sr}_{0.95} \mathrm{La}_{0.05} \mathrm{TiO}_{3}$ should be set at least $200 \AA$.

\section{Acknowledgements}

The present numerical study was performed based on the XFH experiment performed at BL09XU of SPring-8 (Proposal No. 2013B1368).
References

[1] K. Hayashi, N. Uchitomi, J. T. Asubar, N. Happo, W. Hu, S. Hosokawa, and M. Suzuki, Jpn. J. Appl. Phys., 50, 01BF05-1-4 (2011).

[2] W. Hu, K. Hayashi, T. Fukumura, K. Akagi, M. Tsukada, N. Happo, S. Hosokawa, K. Ohwada, M. Takahasi, M. Suzuki, and M. Kawasaki, Appl. Phys. Lett., 106, 222403-1-5 (2015).

[3] T. Fujiwara, L. An, Y. Park, N. Happo, K. Hayashi, and H. Onishi, Thin Solid Films, 658, 66-72 (2018).

[4] Y. Ebisu, K. Hayashi, N. Happo, S. Hosokawa, and T. Ozaki, Trans. Mat. Res. Soc. Japan, 40, 355-358 (2015). https://doi.org/10.14723/tmrsj.40.355

[5] K. Kimura, K. Hayashi, L. V. Yashina, N. Happo, T. Nishioka, Y. Yamamoto, Y. Ebisu, T. Ozaki, S. Hosokawa, T. Matsushita, and H. Tajiri, Surf. Interface Anal., 51, 51-55 (2019).

[6] J. J. Barton, Phys. Rev. Lett., 67, 3106-3109 (1991).

[7] S. Hosokawa, N. Happo, T. Ozaki, H. Ikemoto, T. Shishido, and K. Hayashi, Phys. Rev. B, 87, 094104-1-8 (2013).

[8] K. Hayashi, N. Happo, and S. Hosokawa, J. Electron. Spectrosc. Relat. Phenom., 195, 337-346 (2014).

[9] N. Happo, K. Hayashi, S. Senba, H. Sato, M. Suzuki, and S. Hosokawa, J. Phys. Soc. Jpn., 83, 113601-1-4 (2014).

[10] K. Hayashi, N. Uchitomi, K. Yamagami, A. Suzuki, H. Yoshizawa, J. T. Asubar, N. Happo, and S. Hosokawa, J. Appl. Phys., 119, 125703-1-9 (2016).

[11] T, Matsushita, e-J. Surf. Sci. Nanotech., 14, 158-160 (2016).

[12] T. Yamamoto, K. Hayashi, N. Happo, S. Hosokawa, and H. Tajiri, Acta Mater., 131, 534-542 (2017).

[13] Y. Nishino, T. Ishikawa, K. Hayashi, Y. Takahashi, and E. Matsubara, Phys. Rev. B, 66, 092105-1-4 (2002).

(Received December 27, 2018; Accepted February 19, 2019; Published Online April 1, 2019) 